DRAFT VERSION JULY 5, 2021

Preprint typeset using $\mathrm{LT}_{\mathrm{E}} \mathrm{X}$ style emulateapj v. 12/16/11

\title{
CANDELS SHEDS LIGHT ON THE ENVIRONMENTAL QUENCHING OF LOW-MASS GALAXIES
}

\author{
Yicheng Guo $^{1}$, Eric F. Bell ${ }^{2}$, Yu Lu ${ }^{3}$, David C. Koo ${ }^{1}$, S. M. FAber ${ }^{1}$, Anton M. Koekemoer ${ }^{4}$, Peter Kurczynski ${ }^{5}$,

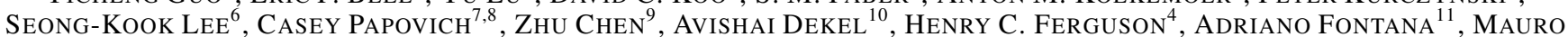

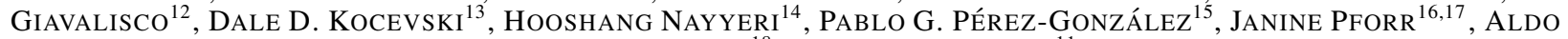 \\ RODRÍGUEZ-PUEBLA ${ }^{18}$, AND PAOLA SANTINI ${ }^{11}$ \\ Draft version July 5, 2021
}

\begin{abstract}
We investigate the environmental quenching of galaxies, especially those with stellar masses $\left(M_{*}\right)<$ $10^{9.5} \mathrm{M}_{\odot}$, beyond the local universe. Essentially all local low-mass quenched galaxies (QGs) are believed to live close to massive central galaxies, which is a demonstration of environmental quenching. We use CANDELS data to test whether or not such a dwarf QG-massive central galaxy connection exists beyond the local universe. For this purpose, we only need a statistically representative, rather than a complete, sample of low-mass galaxies, which enables our study to $z \gtrsim 1.5$. For each low-mass galaxy, we measure the projected distance $\left(d_{\text {proj }}\right)$ to its nearest massive neighbor $\left(\mathrm{M}_{*}>10^{10.5} \mathrm{M}_{\odot}\right)$ within a redshift range. At a given $z$ and $\mathrm{M}_{*}$, the environmental quenching effect is considered to be observed if the $d_{\text {proj }}$ distribution of QGs $\left(d_{\text {proj }}^{Q}\right)$ is significantly skewed toward lower values than that of star-forming galaxies $\left(d_{p r o j}^{S F}\right)$. For galaxies with $10^{8} \mathrm{M}_{\odot}<\mathrm{M}_{*}<10^{10} \mathrm{M}_{\odot}$, such a difference between $d_{\text {proj }}^{Q}$ and $d_{\text {proj }}^{S F}$ is detected up to $z \sim 1$. Also, about $10 \%$ of the quenched galaxies in our sample are located between two and four virial radii $\left(R_{V i r}\right)$ of the massive halos. The median projected distance from low-mass QGs to their massive neighbors, $d_{p r o j}^{Q} / R_{V i r}$, decreases with satellite $\mathrm{M}_{*}$ at $\mathrm{M}_{*} \lesssim 10^{9.5} \mathrm{M}_{\odot}$, but increases with satellite $\mathrm{M}_{*}$ at $\mathrm{M}_{*} \gtrsim 10^{9.5} \mathrm{M}_{\odot}$. This trend suggests a smooth, if any, transition of the quenching timescale around $\mathrm{M}_{*} \sim 10^{9.5} \mathrm{M}_{\odot}$ at $0.5<z<1.0$.
\end{abstract}

\section{INTRODUCTION}

Environmental effects are believed to be the primary process of ceasing star formation in low-mass galaxies with stellar masses $\left(\mathrm{M}_{*}\right)$ lower than $10^{9.5} \mathrm{M}_{\odot}$ (or dwarf galaxies). Field low-mass galaxies may temporarily quench their star

\footnotetext{
${ }^{1} \mathrm{UCO} /$ Lick Observatory, Department of Astronomy and Astrophysics, University of California, Santa Cruz, CA, USA; ycguo@ucolick.org

2 Department of Astronomy, University of Michigan, Ann Arbor, MI, USA

${ }^{3}$ Observatories, Carnegie Institution for Science, Pasadena, CA, USA

${ }^{4}$ Space Telescope Science Institute, Baltimore, MD, USA

${ }^{5}$ Department of Physics and Astronomy, Rutgers University, Piscataway, NJ, USA

${ }^{6}$ Center for the Exploration of the Origin of the Universe, Department of Physics and Astronomy, Seoul National University, Seoul, Korea

${ }^{7}$ Department of Physics and Astronomy, Texas A\&M University, College Station, TX, USA

${ }^{8}$ George P. and Cynthia Woods Mitchell Institute for Fundamental Physics and Astronomy, Texas A\&M University, College Station, TX, USA

${ }^{9}$ Shanghai Key Lab for Astrophysics, Shanghai Normal University, 100 Guilin Road, 200234, Shanghai, China

${ }^{10}$ Center for Astrophysics and Planetary Science, Racah Institute of Physics, The Hebrew University, Jerusalem, Israel

${ }^{11}$ INAF-Osservatorio Astronomico di Roma, Via Frascati 33, I-00078 Monte Porzio Catone, Rome, Italy

12 Department of Astronomy, University of Massachusetts, Amherst, MA, USA

${ }^{13}$ Colby College, Waterville, ME, USA

${ }^{14}$ Department of Physics and Astronomy, University of California, Irvine, CA, USA

${ }^{15}$ Departamento de Astrofísica, Facultad de CC. Físicas, Universidad Complutense de Madrid, E-28040 Madrid, Spain

16 Aix Marseille Université, CNRS, LAM (Laboratoire d'Astrophysique de Marseille) UMR 7326, 13388, Marseille, France

${ }^{17}$ ESA/ESTEC, Noordwijk, The Netherlands

${ }^{18}$ Instituto de Astronomía, Universidad Nacional Autónoma de México, A. P. 70-264, 04510 México, D.F., México
}

formation through supernova feedback, but new gas accretion and recycling would induce new starbursts with periods of tens of megayears (e.g., Guo et al. 2016; Sparre et al. 2017). Geha et al. (2012, hereafter G12) found that the quenched fraction of galaxies with $\mathrm{M}_{*}<10^{9} \mathrm{M}_{\odot}$ drops rapidly as a function of distance to massive host galaxies and that essentially all local field galaxies in this mass regime are forming stars.

The environmental quenching of low-mass galaxies beyond the local universe, however, is rarely investigated because of these galaxies' faint luminosity. Most studies (e.g., G12; Ouadri et al. 2012; Tal et al. 2013, 2014; Balogh et al. 2016; Kawinwanichakij et al. 2016; Fossati et al. 2017, etc.) start from central galaxies and measure the quenched fraction of their satellites. This method requires a complete sample of satellites, which limits these studies to the local universe and/or to intermediate-mass $\left(\mathrm{M}_{*} \geq 10^{9.5} \mathrm{M}_{\odot}\right)$ satellites.

In this letter, we use CANDELS data (Grogin et al. 2011; Koekemoer et al. 2011) to detect the effects of environmental quenching beyond $z \sim 1$. Our approach is different from but complementary to other studies. We start from the "victims" - quenched dwarf galaxies — and search for their massive neighbors, which are tracers of massive dark matter halos.

The concept of our approach is simple - if environmental effects are solely responsible for quenching all dwarf galaxies, all low-mass quenched galaxies (QGs) should live close to a massive central galaxy in a massive halo. In contrast, star-forming galaxies (SFGs) can live far away from massive dark matter halos. Therefore, on average, QGs should have systematically shorter distances to their massive neighbors than SFGs should. This systematic difference between the two populations is evidence of the dwarf QG-massive central galaxy connection and therefore a demonstration of environmental quenching. Because our goal is to investigate whether 
or not such a dwarf QG-massive central connection has been established, we only need to detect a statistically meaningful signal, rather than to find all signals, to rule out the null hypothesis of no environmental effects. This advantage allows us to use an incomplete dwarf sample to study this topic beyond the local universe.

We adopt a flat $\Lambda$ CDM cosmology with $\Omega_{m}=0.3, \Omega_{\Lambda}=$ 0.7 , and the Hubble constant $h \equiv H_{0} / 100 \mathrm{~km} \mathrm{~s}^{-1} \mathrm{Mpc}^{-1}=$ 0.70. We use the AB magnitude scale (Oke 1974) and a Chabrier (2003) IMF.

\section{DATA}

We use the photometric redshift (photo-z), $\mathrm{M}_{*}$, and restframe color catalogs of four CANDELS fields: GOODS-S (Guo et al. 2013), UDS (Galametz et al. 2013), GOODS-N (G. Barro et al., in preparation) and COSMOS (Nayyeri et al. 2017).

The photo-z measurement is described in Dahlen et al. (2013). For GOODS-S galaxies at $0.5<z<2.0$ and $H<26$ $\mathrm{AB}$, the $1 \sigma$ scatter of $|\Delta z| /(1+z)$ is 0.026 and the outlier fraction (defined as $|\Delta z| /(1+z)>0.1$ ) is $8.3 \%$. We also divide the test sample into low-mass $\left(\mathrm{M}_{*}<10^{9} \mathrm{M}_{\odot}\right)$ and massive $\left(\mathrm{M}_{*}>10^{9} \mathrm{M}_{\odot}\right)$ sub-samples. The $1 \sigma$ scatter and outlier fraction of the low-mass (and massive) sub-sample are 0.033 (0.024) and $13.7 \%(7.2 \%)$.

The $\mathrm{M}_{*}$ measurement is described in Santini et al. (2015), where each galaxy is fit by 12 SED-fitting codes with different combinations of synthetic stellar population models, star formation histories, fitting methods, etc. For each galaxy, we use the median of the 12 best-fit $M_{*}$ as its $M_{*}$. The typical uncertainty of $M_{*}$ measurement is $\sim 0.15$ dex. Rest-frame colors are measured by using EAZY (Brammer et al. 2008).

\section{METHOD}

\subsection{Sample Selection}

Our sample consists of sources with F160W $H<26$ AB, PHOTFLAG $=0$ (no suspicious photometry), and SExtractor CLASS_STAR $<0.8$. The magnitude limit of $H=26 \mathrm{AB}$ is approximately the $50 \%$ completeness limit of CANDELS wide regions (Guo et al. 2013) and it is corresponding to a galaxy of $\mathrm{M}_{*} \sim 10^{8} \mathrm{M}_{\odot}$ at $z \sim 0.5$ with a single stellar population that is 5 Gyr old. We divided the whole sample into different $z$ and $\mathrm{M}_{*}$ bins: $z=0.5-2$ with $\Delta z=0.25$ and $\log \left(\mathrm{M}_{*}\right)=8.0-10.5$ with $\Delta \log \left(\mathrm{M}_{*}\right)=0.5$.

In each $\left(z, M_{*}\right)$ bin, we use the UVJ diagram (Williams et al. 2009; Muzzin et al. 2013) to select QGs and SFGs. To avoid the contamination of misidentified stars and sources with suspicious colors, we add one criterion to refine the quenching region (the diagonal light brown line within the original UVJ quenched region in Panel (a)s of Figures 1 . 3). This extra criterion may exclude some very compact QGs (Barro et al. 2013), but since our goal is to obtain a clean and statistically meaningful sample rather than a complete one, such exclusion is necessary and does not affect our results.

For SFGs, instead of using all galaxies in the UVJ starforming region, we measure the median and $\pm 1.5 \sigma$ level of the star-forming locus (calculated in the directions parallel and perpendicular to the reddening vector) and use them as the selection boundary. The selected SFGs are plotted as blue points in Panel (a)s of Figures 1 13 . Again, although many galaxies in the original UVJ star-forming locus are excluded, we aim at constructing a clean rather than complete sample.

\subsection{Detecting Environmental Quenching Effects}

For each low-mass galaxy, we search for its nearest massive neighbor in sky (projected distance). The massive sample is selected to have CLASS_STAR $<0.8$, PHOTFLAG $=0, \quad \mathrm{M}_{*}$ massive $>10^{10.5} \mathrm{M}_{\odot}, \quad$ and

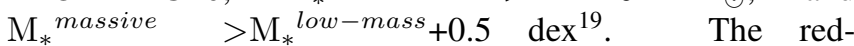
shift range of the massive sample is limited to $\left|z_{\text {massive }}-z_{\text {low-mass }}\right| /\left(1+z_{\text {low-mass }}\right)<0.10$, which is about $3 \sigma$ of our photo-z accuracy. We calculate the projected distances between the low-mass galaxy and the selected massive galaxies. The massive galaxy with the smallest projected distance is chosen as the central galaxy of the low-mass galaxy. We use $d_{\text {proj }}$ to denote this smallest projected distance.

Because of projection effects, a massive neighbor found through this method may not be the real massive galaxy whose dark matter halo was responsible for quenching the low-mass galaxy. But if environmental effects are the primary way of quenching a population of low-mass galaxies, statistically, QGs should be located closer to massive companions than SFGs should. As a result, the $d_{\text {proj }}$ distribution of a quenched population $\left(d_{p r o j}^{Q}\right)$ should be skewed toward lower values than that of SFGs $\left(d_{p r o j}^{S F}\right)$.

Many studies (e.g., Scoville et al. 2013; Davies et al. 2016) used the local overdensity field constructed by Voronoi tessellation or the nearest neighbor method to measure environments. Since the local overdensity of a satellite galaxy is correlated with $d_{p r o j}$, our method is similar to those using a density field. While our simple method provides necessary information to test the whether or not question of our particular interest, future work with the density field approach could provide more accurate and detailed measurements of environmental quenching.

We test whether $d_{p r o j}^{Q}$ is systematically and significantly smaller than $d_{\text {proj }}^{S F}$ in each $\left(z, \mathrm{M}_{*}\right)$ bin. Because in most $(z$, $\mathrm{M}_{*}$ ) bins, the number of QGs is much smaller than that of SFGs, the small number statistics needs to be taken into account. In each of these bins, we randomly draw a sub-sample of the SFGs to match the number of the QGs and calculate the median, probability distribution function (PDF), and cumulative distribution functions (CDF) of $d_{\text {proj }}$ of the sub-sample $\left(d_{p r o j}^{S F, s u b}\right)$. We repeat this bootstrapping sampling 3000 times, obtaining 3000 distributions of $d_{p r o j}^{S F, s u b}$. To exclude the null hypothesis, we ask the median $d_{p r o j}^{Q}$ to be $3 \sigma$ smaller than the median of $d_{p r o j}^{S F, s u b}$.

Panels (b) in Figures 13 show some examples of our results. The $\left(z, \mathrm{M}_{*}\right)$ bins labeled with $\geq 3 \sigma$ values (i.e., median $d_{p r o j}^{Q}$ is $3 \sigma$ smaller than median $d_{p r o j}^{S \bar{F}, s u b}$ ) are considered to have an established quenching-environment connection. In contrast, bins with $<3 \sigma$ values cannot rule out the null hypothesis of the two populations having the same $d_{\text {proj }}$ distributions with $3 \sigma$ confidence.

19 The last requirement only affects galaxies with $10^{10} \mathrm{M}_{\odot}<\mathrm{M}_{*}$ low-mass $<10^{10.5} \mathrm{M}_{\odot}$. The $\mathrm{M}_{*}$ massive threshold of our massive sample corresponds to dark matter halos of $\mathrm{M}_{\text {halo }} \gtrsim 10^{12} \mathrm{M}_{\odot}$. Since the $M_{*}-M_{\text {halo }}$ relation evolves little with redshift in this mass regime (Behroozi et al. 2013), our choice of a fixed $\mathrm{M}_{*}$ massive threshold at different redshifts allows us to investigate the environmental effects of similar $\mathrm{M}_{\text {halo }}$ at different cosmic times. 

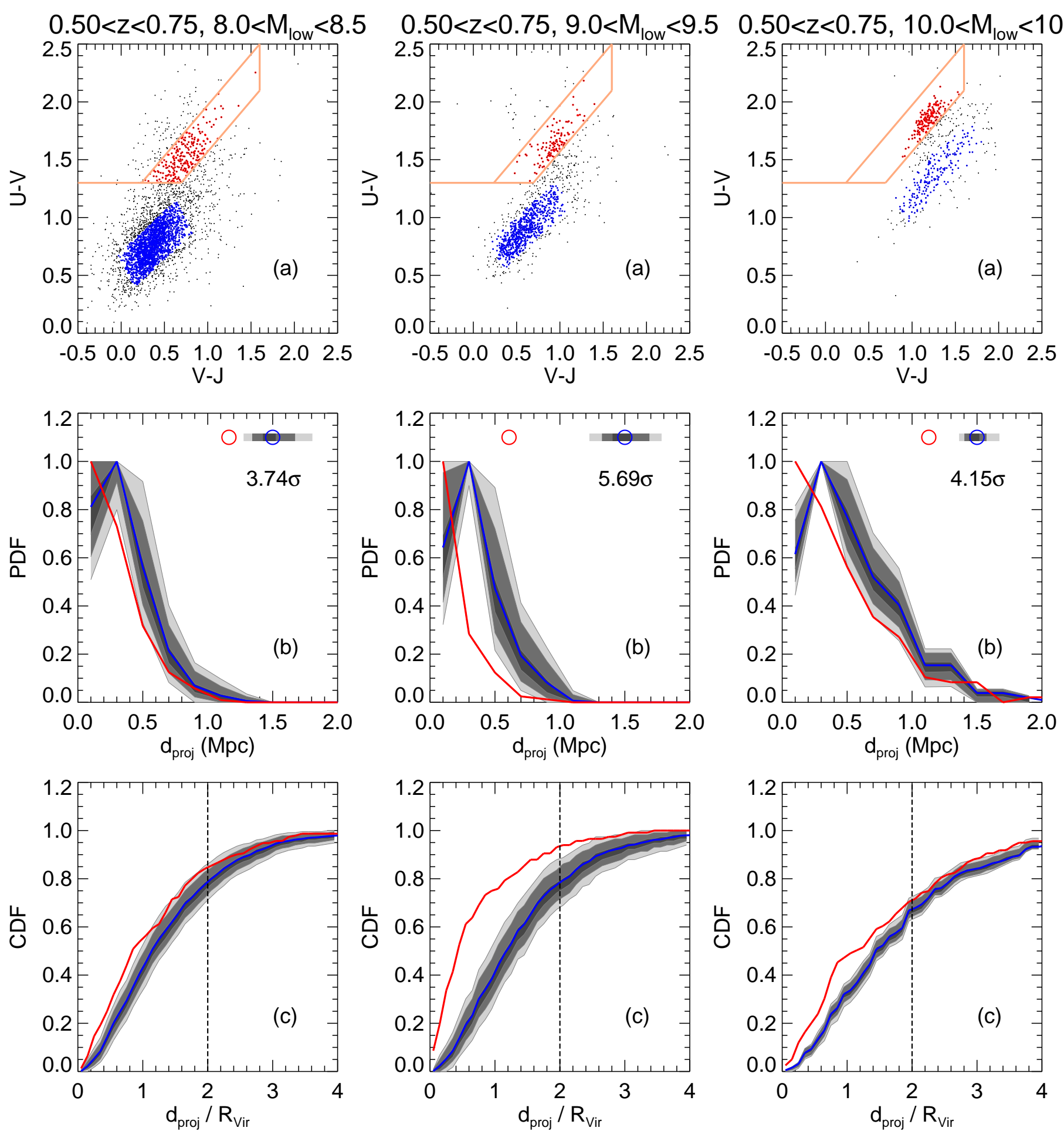

FIG. 1.- Examples of sample selection and environment measurement. Each column shows a given $\left(z, \mathrm{M}_{*}\right)$ bin as the title shows. In each column, Panel (a) shows the selected QGs (red) and SFGs (blue) in the UVJ diagram. Black dots show all galaxies (with $H_{F 160 W}<26$ and CLASS_STAR $<0.8$ ) in this bin. Panel (b) shows the PDFs of $d_{p r o j}$ of the QGs (red) and SFGs (blue) galaxies. The dark, medium, and light gray regions show the $1 \sigma, 2 \sigma$, and $3 \sigma$ levels of 3000 times of bootstrapping of SFGs to match the number of the QGs. The red and blue circles show the medians of $d_{p r o j}$ of the QGs and SFGs. The gray bar shows the $1 \sigma$ (dark), $2 \sigma$ (medium), and $3 \sigma$ (light) levels of the medians of the bootstrapping. To show the difference clearly, all median values are normalized so that the median of the SFGs (blue circle) is equal to $1.5 \mathrm{Mpc}$. The number below the gray bar shows the confidence level to which the null hypothesis that the QGs (red) and SFGs (blue) have the same $d_{\text {proj }}$ medians is ruled out. Panel (c) shows the CDFs of $d_{\text {proj }}$ of the QGs (red) and SFGs (blue) normalized by $R_{V i r}$ of the halos of their massive neighbors. The dark, medium, and light gray regions show the $1 \sigma, 2 \sigma$, and $3 \sigma$ levels of the bootstrapping. All columns in this figure are at $0.5<z<0.75$. 

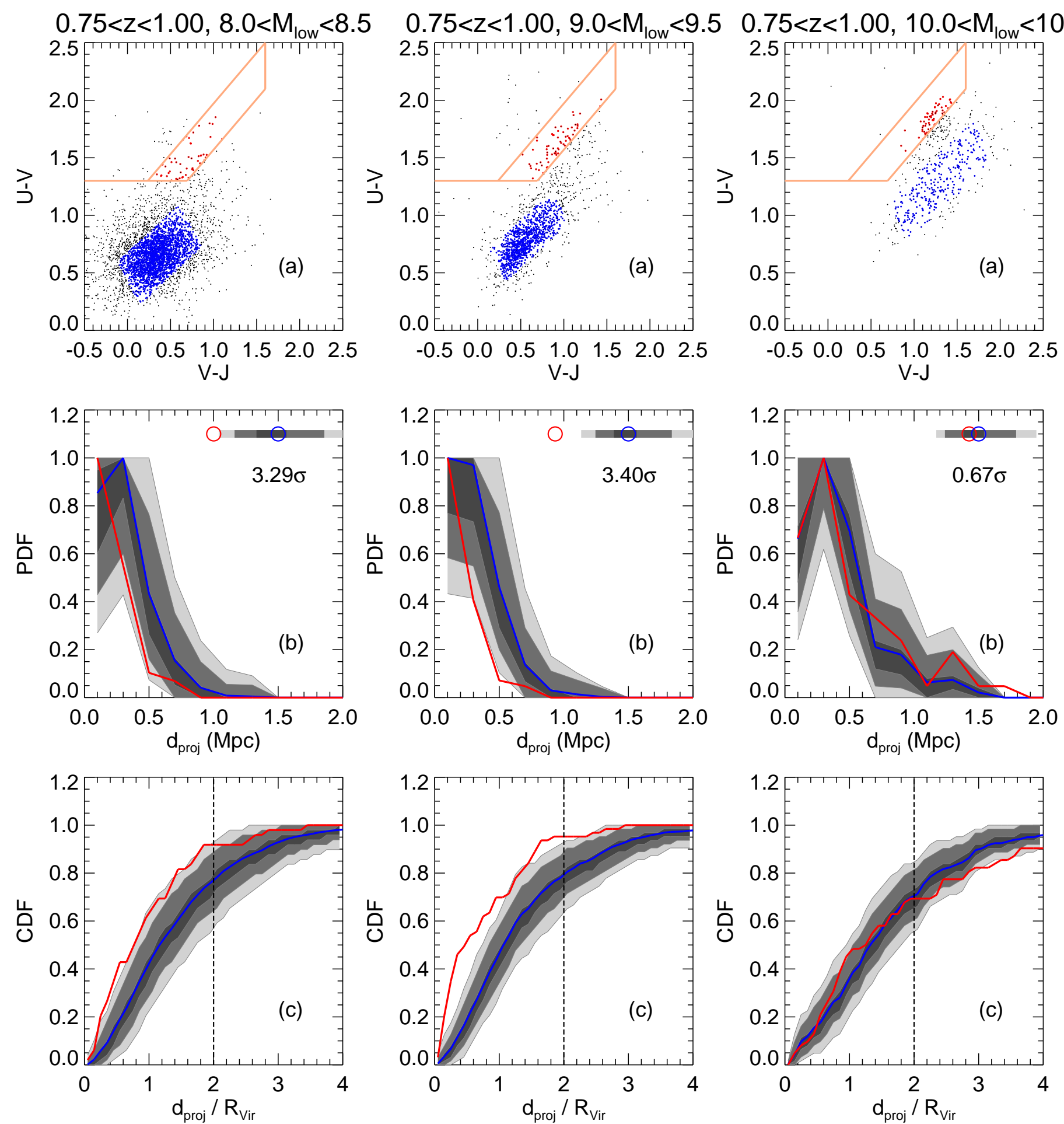

FIG. 2.- Same as Figure 1 but showing three $\mathrm{M}_{*}$ bins at $0.75<z<1.00$. 

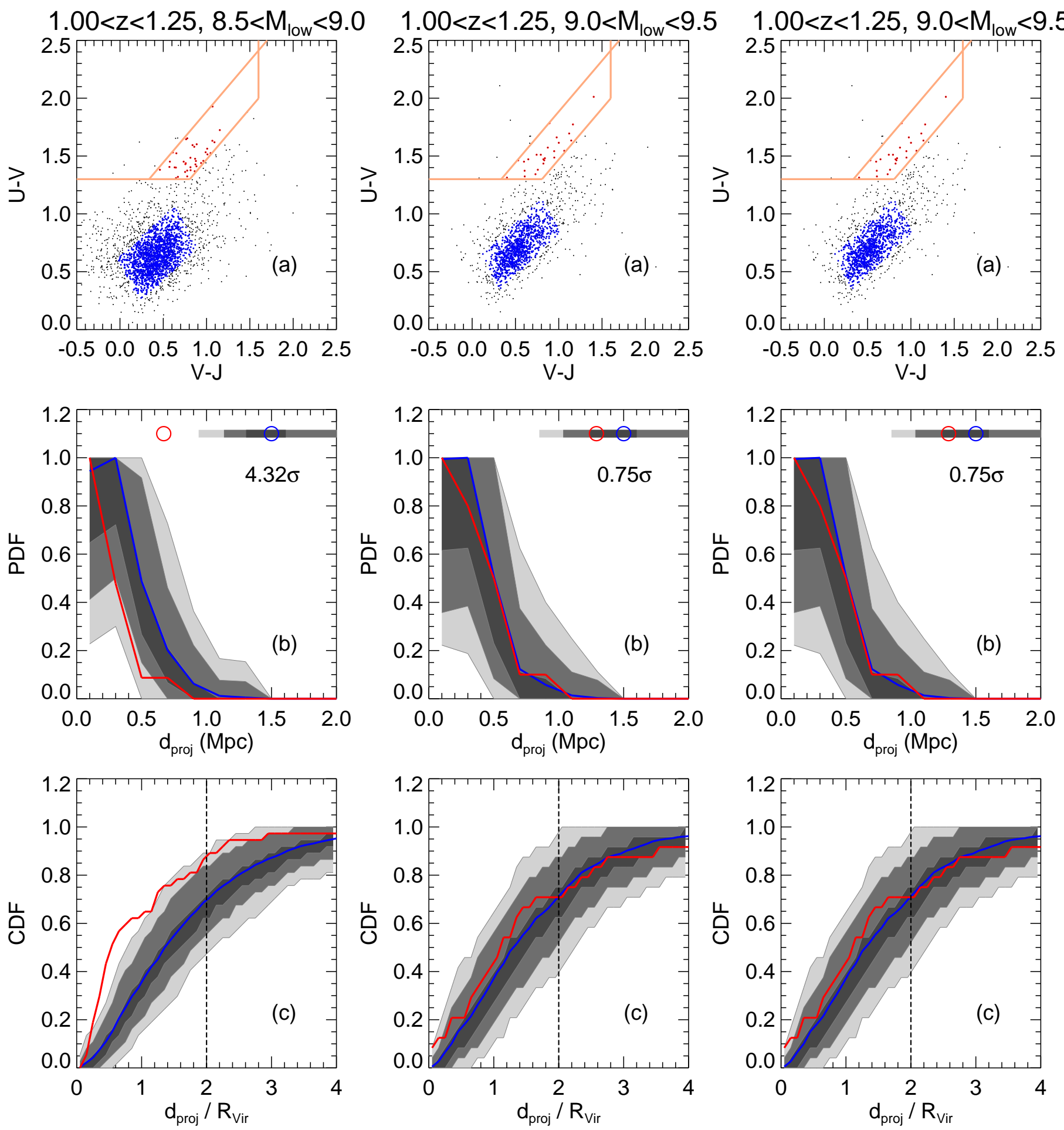

FIG. 3. - Same as Figure 1 but showing three $\mathrm{M}_{*}$ bins at $1.00<z<1.25$.

\section{RESULTS}

Panel (a) of Figure 4 shows the deviation from $d_{\text {proj }}^{Q}$ to $d_{\text {proj }}^{S F, s u b}$ in each $\left(z, \mathrm{M}_{*}\right)$ bin. Our criterion of environmental quenching being observed is that the median of $d_{p r o j}^{Q}$ is $3 \sigma$ smaller than that of $d_{\text {proj }}^{S F, \text { sub }}$. For galaxies with $10^{8} \mathrm{M}_{\odot}<\mathrm{M}_{*}<$ $10^{10} \mathrm{M}_{\odot}$, such a quenching-environment connection is ob- served up to $z \sim 1$, as shown by the larger-than-3 $\sigma$ deviations. This result is consistent with the quick emergence of low-mass QGs from the measurement of stellar mass functions at $z \sim 1$ (e.g., Ilbert et al. 2013; Huang et al. 2013). For galaxies with $10^{10.0} \mathrm{M}_{\odot}<\mathrm{M}_{*}<10^{10.5} \mathrm{M}_{\odot}$, the connection was established at a lower redshift.

For those $\left(z, \mathrm{M}_{*}\right)$ bins with $<3 \sigma$ deviation, we cannot rule out the null hypothesis of QGs and SFGs having the 

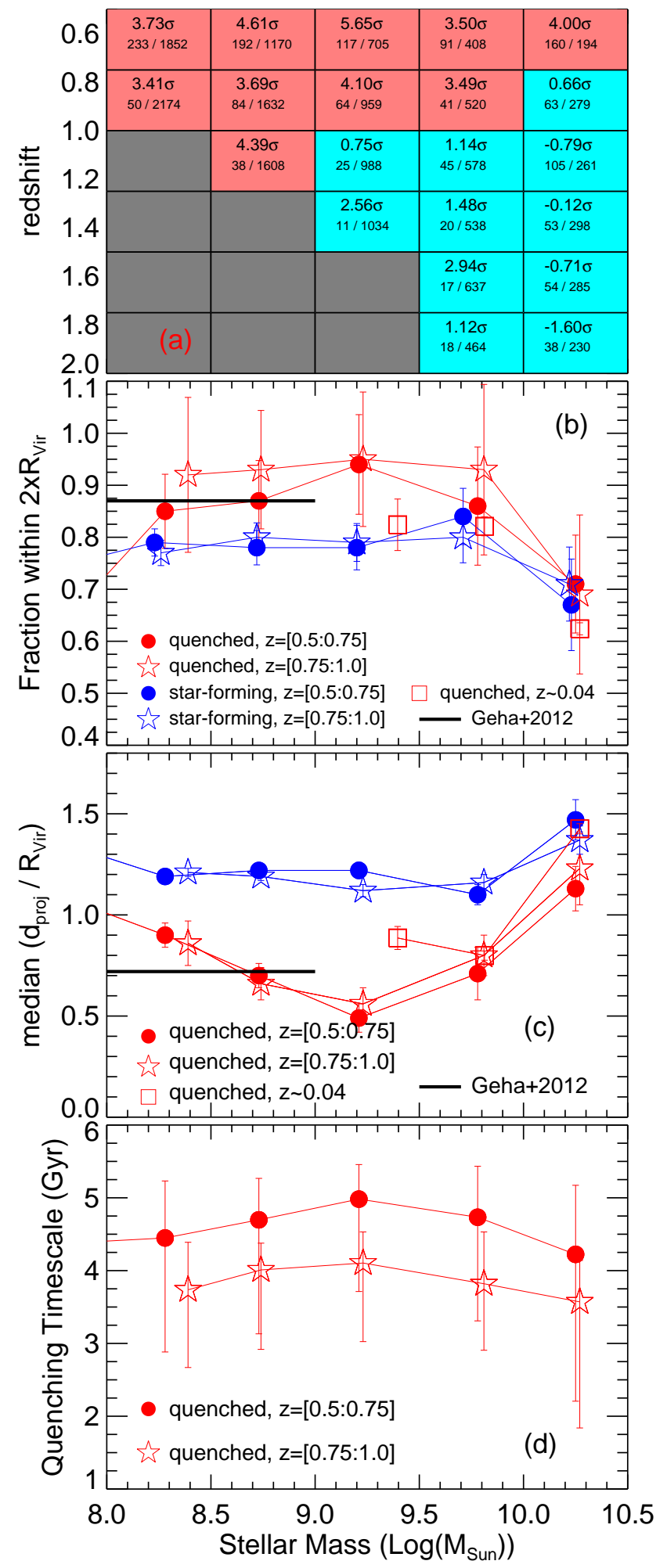

FIG. 4._Panel (a): Statistics of the quenching-environment connection. In each $\left(z, \mathrm{M}_{*}\right)$ bin, the deviation between the medians of $d_{p r o j}^{Q}$ and $d_{\text {proj }}^{S F, s u b}$ is the upper number, while the numbers of the QGs and SFGs are the two lower numbers. We choose the deviation $\geq 3 \sigma$ as the threshold of the quenchingenvironment connection being observed. All such bins are red, while others with the deviation $<3 \sigma$ are cyan. Gray bins cannot be accessed by our current dataset. Panel $(b)$ : Fraction of a population of galaxies within $2 R_{V \text { ir }}$ of massive halos. Different symbols show different populations. Panel (c): Median $d_{\text {proj }} / R_{V i r}$ of different samples. Panel $(d)$ : Inferred quenching timescales in two redshift bins. The colors and symbols in Panel (b), (c), and (d) are the same.
Guo et al.
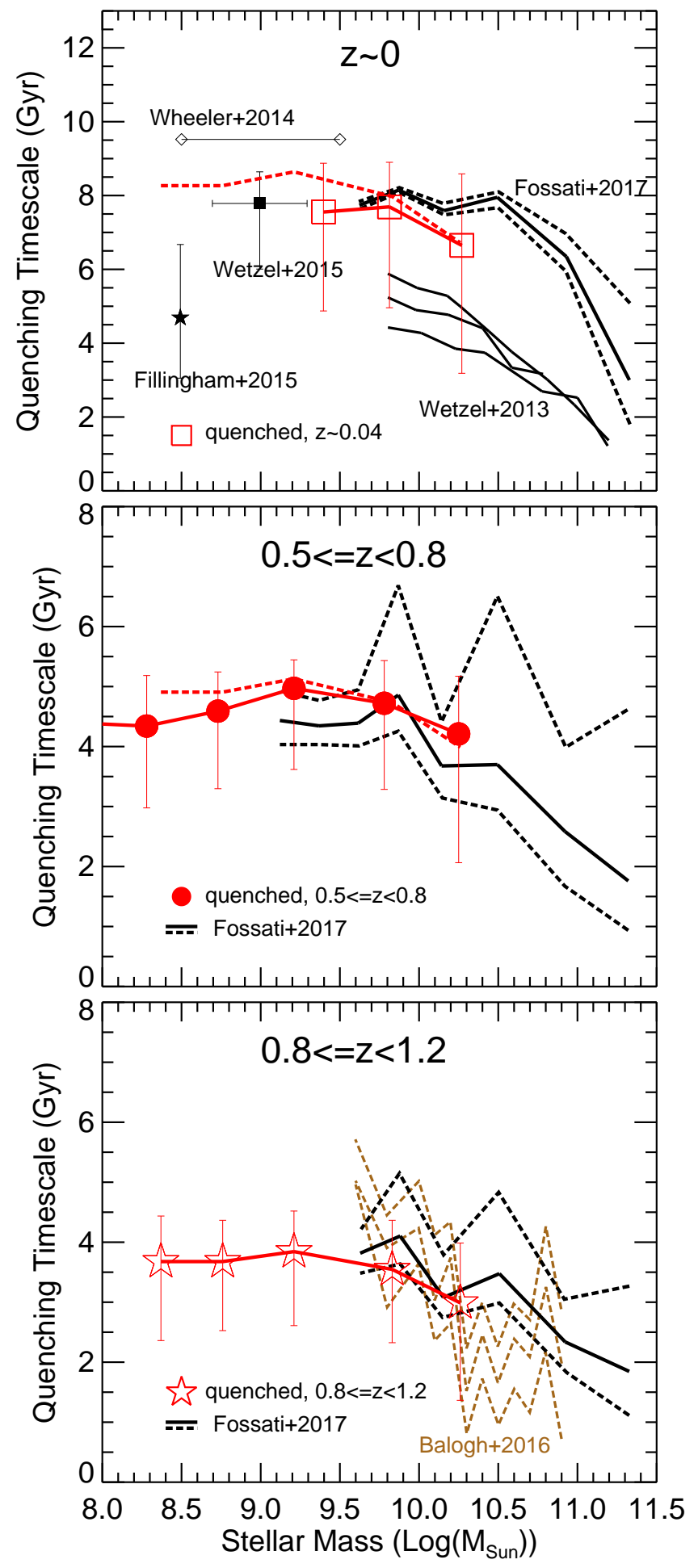

FIG. 5.- Quenching timescale at different redshifts. The red symbols are calculated by this work, while the black and brown lines and symbols are taken from the literature. The red dashed lines in the first two panels are the solid red line in the third panel (i.e., $T_{Q}$ at $0.8 \leq z<1.2$ ) scaled up by $(1+z)^{1.5}$.

same $d_{\text {proj }}$ distributions with more than $3 \sigma$ confidence. This may imply that the quenching-environment connection has not been established in these bins. This interpretation is at least consistent with some other studies for massive galaxies $\left(\mathrm{M}_{*}>10^{10} \mathrm{M}_{\odot}\right)$, which claimed that massive quiescent galax- 
ies at $z>1$ are not necessarily located in high-density environments (e.g., Darvish et al. 2015, 2016; Lin et al. 2016). For lower-mass galaxies at $z \gtrsim 1.5$, however, due to projection effects or small number statistics, our method may have failed to detect existing quenching-environment connections.

\section{DISCUSSION}

\subsection{Spatial Distribution of Quenched Galaxies}

G12 found that $87 \%$ (and 97\%) of dwarf QGs in their SDSS sample are within $2 R_{V i r}$ (and $4 R_{V i r}$ ) of a massive host galaxy. We find similar results in our sample at $0.5<z<1.0$ (Panel (b) of Figure 4). About $90 \%$ of the QGs below $10^{10} \mathrm{M}_{\odot}$ in our sample are within $2 R_{V i r}$. The fraction drops quickly to about $70 \%$ for galaxies above $10^{10} \mathrm{M}_{\odot}$. To calculate $R_{V i r}$, we first use the $\mathrm{M}_{*}-\mathrm{M}_{\text {halo }}$ relation of Behroozi et al. (2013) to obtain $\mathrm{M}_{\text {halo }}\left(M_{\mathrm{vir}}\right)$ of the massive neighbors. Then, we derive $R_{V i r}$ through $M_{\mathrm{vir}}(z)=$ $\frac{4 \pi}{3} \Delta_{\mathrm{c}}(z) \rho_{\text {crit }}(z) R_{\text {vir }}(z)^{3}$, where $\rho_{\text {crit }}(z)$ is the critical density of the universe at $z$, and $\Delta_{\mathrm{C}}(z)$ is calculated by following Bryan \& Norman (1998).

SFGs in our sample are also almost within $4 R_{V i r}$. This could be a projection effect. Since we search for massive neighbors within a long line-of-sight distance $(|\Delta z| /(1+z)<$ $0.10)$, an SFG has a high chance of being located within the projected $4 R_{V i r}$ of a massive galaxy, even though the massive galaxy is not its real central galaxy. In contrast, with a more accurate redshift measurement, G12 found that only $\sim 50 \%$ of the $z \sim 0$ SFGs are within $4 R_{V i r}$, suggesting that a large fraction of SFGs are intrinsically outside $4 R_{V i r}$. QGs in our sample may suffer from the same projection effect. However, as discussed in Section 3.2 this effect would not affect our statistical results.

A non-negligible fraction (10\%) of low-mass QGs are located between 2 and $4 R_{V i r}$. They are likely central galaxies quenched by mechanisms not related to environment, e.g., AGN and stellar feedback. They, however, may also be evidence for quenching processes acting at large distances of massive halos (e.g., Cen 2014). Y. Lu et al. (2017, in preparation) found that, to match the observed $M_{*}$ and stellar-phase metallicity simultaneously, gas accretion of Milky Way (MW) satellite galaxies need to be largely reduced way before they fall into $R_{V i r}$ of the MW halo, possibly by heating up the intergalactic medium in the MW halo vicinity to $10^{5} \mathrm{~K}$. Alternatively, Slater \& Bell (2013) used simulations to show that environmental effects are prominent out to $2-3 R_{V i r}$ : satellites with very distant apocenters can be quenched by tidal stripping and ram pressure stripping following a close passage to the host galaxy.

We also extend the local results of G12 to higher $M_{*}$ by repeating our measurements on the SDSS sample of Rodríguez-Puebla et al. (2015). The results (red squares in Panel (b) of Figure 4), together with G12, suggest that the fraction of QGs within $2 R_{V i r}$ has almost no redshift dependence. This constant fraction suggests that, at all redshifts, environment (especially within $2 R_{V i r}$ ) dominates the quenching of low-mass galaxies.

We also study the median distance of galaxies to their massive neighbors scaled by $R_{V i r}$ (Panel (c) of Figure 4). SFGs have a constant median distance of $\sim 1.3 R_{V i r}$ over a wide $\mathrm{M}_{*}$ range. QGs are closer to massive neighbors, but their median distance depends on $\mathrm{M}_{*}$ : it decreases from $1 R_{V i r}$ at $10^{8} \mathrm{M}_{\odot}$ to $0.5 R_{V i r}$ at $10^{9.5} \mathrm{M}_{\odot}$, then increases to $>1 R_{V i r}$ at $10^{10.5} \mathrm{M}_{\odot}$. Also, we find no significant difference between different redshifts.

\subsection{Quenching Timescale}

Quenching timescale $\left(T_{Q}\right)$ is important to constrain quenching mechanisms. In the local universe, at $\mathrm{M}_{*}>$ $10^{10} \mathrm{M}_{\odot}$, quenching likely occurs through starvation, whose timescale (4-6 Gyr) is comparable to gas depletion timescales (Fillingham et al. 2015; Peng et al. 2015). At $\mathrm{M}_{*}<10^{8} \mathrm{M}_{\odot}$, ram pressure stripping is likely the dominant mechanism (Slater \& Bell 2014; Fillingham et al. 2015; Weisz et al. 2015). Its timescale (2 Gyr) is much shorter and comparable to the dynamical timescale of the host dark matter halo. The dominant quenching mechanism may change around a characteristic $\mathrm{M}_{*}$.

To infer $T_{Q}$, we assume all galaxies start quenching at 4 $R_{V i r}$. We choose $4 R_{V i r}$ because G12 shows that beyond 4 $R_{V i r}$ the fraction of QGs is almost zero, while the fraction of SFGs is still high. Theoretically, Cen (2014) also predicted the onset of quenching at a similar large halo distance. Galaxies fall into massive halos while their star formation rates are being reduced. They become fully quenched when they arrive at the observed location. Therefore, $T_{Q}$ is the time they spent on traveling from $4 R_{V i r}$ to the observed location with an infall velocity (using circular velocity $V(R)=\sqrt{\frac{G M(<R)}{R}}$ at 2 $R_{V i r}$ as an approximation).

Our method of measuring $T_{Q}$ is different from most studies in the literature, e.g., Wetzel et al. (2013); Wheeler et al. (2014); Fillingham et al. (2015); Balogh et al. (2016); Fossati et al. (2017). They used numerical simulations or semi-analytic models to match the basic demographics (e.g., quenched fraction) of QGs. Our method is purely empirical, but relies on the assumptions of the starting and end points (i.e., $4 R_{V i r}$ and the observed location, respectively) of quenching. Our $T_{Q}$ definition, however, characterizes the same physical quantity as other methods, i.e., the timescale upon which satellites must quench following infalling into the vicinity of their massive hosts.

The inferred $T_{Q}$ is shown in Panel (d) of Figure 4, Overall, the $T_{Q}$ dependence on $\mathrm{M}_{*}$ is, if any, very weak between $10^{8}$ and $10^{10} \mathrm{M}_{\odot}$. Lower-redshift galaxies have longer $T_{Q}$, because dynamical timescale decreases with redshift: lower-redshift galaxies need more time to travel the same $d_{\text {proj }}^{Q} / R_{\text {Vir }}$.

Our measurements show excellent agreement with those of Fossati et al. (2017) and Balogh et al. (2016) at $\mathrm{M}_{*}>$ $10^{9.5} \mathrm{M}_{\odot}$ (Figure 5). Fossati et al. (2017) used 3D-HST data (Skelton et al. 2014; Momcheva et al. 2016) to study the environments of galaxies with $\mathrm{M}_{*} \gtrsim 10^{9.5} \mathrm{M}_{\odot}$ in CANDELS fields. Agreement with these detailed studies provides an assurance to our method: although built upon simplified assumptions, it is able to catch the basic physical principles of environmental quenching. Moreover, the good agreement also implies that the projection effect discussed in Section 5.1 does not significantly bias our measurement.

Our results, together with the measurements of Fossati et al. (2017) and Balogh et al. (2016), imply a smooth $T_{Q}$ transition - and hence a quenching mechanism transition - around $\mathrm{M}_{*} \sim$ $10^{9.5} \mathrm{M}_{\odot}$, which is broadly consistent with other studies (e.g., Cybulski et al. 2014; Lee et al. 2015).

At $\mathrm{M}_{*} \gtrsim 10^{10} \mathrm{M}_{\odot}$, starvation is likely to be responsible for environmental quenching (Fillingham et al. 2015). Alternatively, however, these galaxies could actually be centrals or 
recently quenched before becoming satellites. For them, internal mechanisms (e.g., AGN and star formation feedback) are likely dominating the quenching, as demonstrated by the correlation between star formation and internal structures (e.g., central mass density within $1 \mathrm{kpc}$ discussed in Fang et al. (2013); Barro et al. (2017); Woo et al. (2017)).

The quenching mechanisms at $\mathrm{M}_{*}<10^{9.5} \mathrm{M}_{\odot}$ are still uncertain. Our results suggest that $T_{Q}$ mildly increases with $\mathrm{M}_{*}$ at $0.5 \leq z<0.8$. Other studies of the local universe (e.g., Slater \& Bell 2014; Fillingham et al. 2015; Wetzel et al. 2015) suggest a much stronger $\mathrm{M}_{*}$ dependence of $T_{Q}$. For example, Fillingham et al. (2016) argued that $T_{Q}$ drops quickly to $\sim 2$ Gyr for galaxies with $\mathrm{M}_{*} \lesssim 10^{8} \mathrm{M}_{\odot}$ at $z \sim 0$ because of ram pressure stripping.

At $\mathrm{M}_{*}>10^{9.5} \mathrm{M}_{\odot}$, the redshift dependence of $T_{Q}$ can be explained by the change of the dynamical timescale. We scale up $T_{Q}$ at $0.8 \leq z<1.2$ by a factor of $(1+z)^{1.5}$ to account for the redshift dependence of dynamical timescale (see Tinker \& Wetzel (2010)). This scaled $T_{Q}$ (red dashed lines in the first two panels of Figure 5] matches the actual $T_{Q}$ measurements very well at $\mathrm{M}_{*} \gtrsim 10^{9.5} \mathrm{M}_{\odot}$. However, it deviates from the $T_{Q}$ measurements at $\mathrm{M}_{*}<10^{9.0} \mathrm{M}_{\odot}$. At $z \sim 0$ (7 Gyr after $z \sim 1.0$ ), the scaled $T_{Q}$ is significantly larger than the $T_{Q}$ measured by Fillingham et al. (2015). Balogh et al. (2016) also found similar results: at $z \sim 1$, their $T_{Q}$ of galaxies with $\mathrm{M}_{*}<10^{10} \mathrm{M}_{\odot}$ is longer than the $z \sim 0 T_{Q}$ scaled down by $(1+z)^{1.5}$. Future work is needed to more quantitatively determine the redshift dependence of the $T_{Q}$ of lowmass galaxies.

\section{CONCLUSIONS}

CANDELS allows us to investigate evidence of environmental quenching of dwarf galaxies beyond the local universe. At $0.5<z \lesssim 1.0$, we find that for $10^{8} \mathrm{M}_{\odot}<\mathrm{M}_{*}<$ $10^{10} \mathrm{M}_{\odot}$, QGs are significantly closer to their nearest massive companions than SFGs are, demonstrating that environment plays a dominant role in quenching low-mass galaxies. We also find that about $10 \%$ of the QGs in our sample are located between two and four $R_{V i r}$ of the massive halos. The median projected distance from the QGs to their massive neighbors $\left(d_{\text {proj }}^{Q} / R_{V i r}\right)$ decreases with satellite $\mathrm{M}_{*}$ at $\mathrm{M}_{*} \lesssim$ $10^{9.5} \mathrm{M}_{\odot}$, but increases with satellite $\mathrm{M}_{*}$ at $\mathrm{M}_{*} \gtrsim 10^{9.5} \mathrm{M}_{\odot}$. This trend suggests a smooth, if any, transition of $T_{Q}$ around $\mathrm{M}_{*} \sim 10^{9.5} \mathrm{M}_{\odot}$ at $0.5<z<1.0$.

Y.G., D.C.K., and S.M.F. acknowledge support from NSF grant AST-0808133. Support for Program HST-AR-13891 and HST-GO-12060 were provided by NASA through a grant from the Space Telescope Science Institute, operated by the Association of Universities for Research in Astronomy, Incorporated, under NASA contract NAS5-26555. Z.C. acknowledges support from NSFC grants 11403016 \& 11433003.

\section{REFERENCES}

Balogh, M. L., McGee, S. L., Mok, A., et al. 2016, MNRAS, 456, 4364 Barro, G., Faber, S. M., Pérez-González, P. G., et al. 2013, ApJ, 765, 104 Barro, G., Faber, S. M., Koo, D. C., et al. 2017, ApJ, 840, 47

Behroozi, P. S., Wechsler, R. H., \& Conroy, C. 2013, ApJ, 770, 57

Brammer, G. B., van Dokkum, P. G., \& Coppi, P. 2008, ApJ, 686, 1503

Bryan, G. L., \& Norman, M. L. 1998, ApJ, 495, 80

Cen, R. 2014, ApJ, 781, 38

Chabrier, G. 2003, PASP, 115, 763

Cybulski, R., Yun, M. S., Fazio, G. G., \& Gutermuth, R. A. 2014, MNRAS, 439, 3564

Dahlen, T., Mobasher, B., Faber, S. M., et al. 2013, ApJ, 775, 93

Darvish, B., Mobasher, B., Sobral, D., et al. 2016, ApJ, 825, 113

Darvish, B., Mobasher, B., Sobral, D., Scoville, N., \& Aragon-Calvo, M. 2015, ApJ, 805, 121

Davies, L. J. M., Robotham, A. S. G., Driver, S. P., et al. 2016, MNRAS, 455, 4013

Fang, J. J., Faber, S. M., Koo, D. C., \& Dekel, A. 2013, ApJ, 776, 63

Fillingham, S. P., Cooper, M. C., Pace, A. B., et al. 2016, MNRAS, 463, 1916

Fillingham, S. P., Cooper, M. C., Wheeler, C., et al. 2015, MNRAS, 454, 2039

Fossati, M., Wilman, D. J., Mendel, J. T., et al. 2017, ApJ, 835, 153

Galametz, A., Grazian, A., Fontana, A., et al. 2013, ApJS, 206, 10

Geha, M. Blanton, M. R., Yan, R., \& Tinker, J. L. 2012, ApJ, 757, 85

Grogin, N. A., Kocevski, D. D., Faber, S. M., et al. 2011, ApJS, 197, 35

Guo, Y., Ferguson, H. C., Giavalisco, M., et al. 2013, ApJS, 207, 24

Guo, Y., Rafelski, M., Faber, S. M., et al. 2016, ApJ, 833, 37

Huang, J.-S., Faber, S. M., Willmer, C. N. A., et al. 2013, ApJ, 766, 21

Ilbert, O., McCracken, H. J., Le Fèvre, O., et al. 2013, A\&A, 556, A55

Kawinwanichakij, L., Quadri, R. F., Papovich, C., et al. 2016, ApJ, 817, 9

Koekemoer, A. M., Faber, S. M., Ferguson, H. C., et al. 2011, ApJS, 197, 36

Lee, S.-K., Im, M., Kim, J.-W., et al. 2015, ApJ, 810, 90
Lin, L., Capak, P. L., Laigle, C., et al. 2016, ApJ, 817, 97

Momcheva, I. G., Brammer, G. B., van Dokkum, P. G., et al. 2016, ApJS, 225, 27

Muzzin, A., Marchesini, D., Stefanon, M., et al. 2013, ApJ, 777, 18

Nayyeri, H., Hemmati, S., Mobasher, B., et al. 2017, ApJS, 228, 7

Oke, J. B. 1974, ApJS, 27, 21

Peng, Y., Maiolino, R., \& Cochrane, R. 2015, Nature, 521, 192

Quadri, R. F., Williams, R. J., Franx, M., \& Hildebrandt, H. 2012, ApJ, 744, 88

Rodríguez-Puebla, A., Avila-Reese, V., Yang, X., et al. 2015, ApJ, 799, 130

Santini, P., Ferguson, H. C., Fontana, A., et al. 2015, ApJ, 801, 97

Scoville, N., Arnouts, S., Aussel, H., et al. 2013, ApJS, 206, 3

Skelton, R. E., Whitaker, K. E., Momcheva, I. G., et al. 2014, ApJS, 214, 24

Slater, C. T., \& Bell, E. F. 2013, ApJ, 773, 17

Slater, C. T., \& Bell, E. F. 2014, ApJ, 792, 141

Sparre, M., Hayward, C. C., Feldmann, R., et al. 2017, MNRAS, 466, 88

Tal, T., van Dokkum, P. G., Franx, M., et al. 2013, ApJ, 769, 31

Tal, T., Dekel, A., Oesch, P., et al. 2014, ApJ, 789, 164

Tinker, J. L., \& Wetzel, A. R. 2010, ApJ, 719, 88

Weisz, D. R., Dolphin, A. E., Skillman, E. D., et al. 2015, ApJ, 804, 136

Wetzel, A. R., Tinker, J. L., Conroy, C., \& van den Bosch, F. C. 2013, MNRAS, 432, 336

Wetzel, A. R., Tollerud, E. J., \& Weisz, D. R. 2015, ApJ, 808, L27

Wheeler, C., Phillips, J. I., Cooper, M. C., Boylan-Kolchin, M., \& Bullock, J. S. 2014, MNRAS, 442, 1396

Williams, R. J., Quadri, R. F., Franx, M., van Dokkum, P., \& Labbé, I. 2009. ApJ, 691, 1879

Woo, J., Carollo, C. M., Faber, S. M., Dekel, A., \& Tacchella, S. 2017, MNRAS, 464, 1077 